\title{
2019 Wilderness E Environmental Medicine Peer Reviewers
}

The editors express their sincere appreciation to the following individuals who performed peer reviews for articles submitted to Wilderness \& Environmental Medicine in 2019. WEM serves an important role in bringing thoughtfully reviewed literature to the scientific community. Our peer reviewers play an essential role in ensuring the merit and quality of the manuscripts we publish. Many of these individuals reviewed multiple papers, and some also serve on the editorial board and maintain Section Editor or Associate Editor duties.

(Notes: * WEM editorial board member; $†$ WEM Reviewer-in-Training.)

\author{
Robert Alunday, USA \\ Zuhair Amr, Jordan \\ John Anderson, USA \\ Eldon Askew, USA \\ Chad A. Asplund, USA \\ Henrik Constantin Bäcker, USA \\ Howard Backer, USA* \\ Gina Badalato, USA \\ Jennifer Baine, USA \\ Katarzyna Bartosik, Poland \\ Buddha Basnyat, Nepal* \\ Himmatrao S. Bawaskar, India \\ Brad L. Bennett, USA* \\ Michael Bergeron, USA \\ Jeffrey Bernstein, USA \\ Claire Berryman, USA \\ Sanjeeb Sudarshan Bhandari, USA ${ }^{\dagger}$ \\ Amy Biondich, USA \\ Cindy Carol Bitter, USA \\ Adolfo Borges, Venezuela \\ Ralph Stanbery Bovard, USA \\ Benjamin Earl Brackett, USA ${ }^{\dagger}$ \\ Graham Brant-Zawadzki, USA ${ }^{\dagger}$ \\ Marc Elliott Breen, USA \\ Robert Brickley, $\mathrm{USA}^{\dagger}$ \\ Aaron Brillhart, USA \\ Monika Maria Brodmann Maeder, \\ Switzerland \\ Emma Browne, UK \\ Timothy Brox, USA \\ Hermann Brugger, Italy \\ Keith Burgess, Australia \\ Omer Lee Burnett, USA \\ Martin Burtscher, Austria \\ Gabriel Cade, USA \\ Aaron D. Campbell, USA* \\ Michael Cardwell, USA \\ Jim Caruso, USA \\ John W. Castellani, USA \\ Michael Caudell, USA*
}

Nisha Charkoudian, USA

Ivy Cheng, Canada

Stephen Cheung, Canada

Richard F. Clark, USA

Kirsten Coffman, USA

Sheri R. Colberg, USA

Tyler Colesar, USA ${ }^{\dagger}$

Ed Cornell, USA

Patricia Corrin, Canada

John S. Cuddy, USA

Michael Dacre, USA

Daniel Danzl, USA

Tomasz Darocha, Poland

David DeGroot, USA

Thomas DeLoughery, USA

Petar J. Denoble, USA

James H. Diaz, USA

Brendon Drew, USA

Charles Dumke, USA

Ola Halina Dunin-Bell, Canada

Liz Edelstein, USA

Kurt Power Eifling, USA

Brandon Elder, USA ${ }^{\dagger}$

John Ellerton, UK

Brett Romano Ely, USA

Preston J. Fedor, USA

Francesco Feletti, Italy

Joseph Derek Forrester, USA

Francisco Franca, Brazil

Luanne Freer, USA

Hin Tat T. Fung, China

Arun Ganti, USA ${ }^{\dagger}$

Flavio G. Gaudio, USA

Charles J. Gerardo, USA

Gordon G. Giesbrecht, Canada

Milton Glatterer, USA

Torrey Lisa Goodman, USA

Daniel A. Gragert, USA ${ }^{\dagger}$

Steve Green, USA ${ }^{\dagger}$

Mike Greene, UK
Mark Greve, USA

Justin Grisham, USA ${ }^{\dagger}$

Jay Gupta, USA

Peter Hackett, USA*

Vidal Haddad Jr., Brazil

Stanley Hassinger, USA

Michael Hauty, USA

Tatiana Havryliuk, USA

Seth C. Hawkins, USA

Alana Hawley, USA

Carlton Heine, USA

Mary Josephine Hessert, USA

Natalie Hoelzl, Germany

Ross Hofmeyr, South Africa

Benjamin Honigman, USA

Arthur (Tony) Anthony Islas, USA

Ahmad Khaldun Khaldun Ismail, Malaysia

Bryan Jarrett, USA

Anthony Jiang, USA

Clare Johnson, $\mathrm{USA}^{\dagger}$

Barbara Ellen Jones, USA

Thomas Küpper, Germany

Nicholas Kanaan, USA

Lee Kaplan, USA

Tao Ke, USA ${ }^{\dagger}$

Robert W. Kenefick, USA*

Linda Keyes, USA

Kenneth W Kizer, USA*

Pranawa Koirala, USA

John F. Kragh, USA

Daniela Lamattina, Argentina

Jordan Lane, $\mathrm{USA}^{\dagger}$

Ricky Langley, USA

Stephanie Lareau, USA

Eric John Lavonas, USA

Alexander Li, USA ${ }^{\dagger}$

Stephen Y Liang, USA ${ }^{\dagger}$

James Lieberman, USA

Benjamin Lischner, Norway 
Colin Little, USA ${ }^{\dagger}$

Lanny Littlejohn, USA

Adam Lund, Canada

Gary Mack, USA

Swaminatha Mahadevan, India

Sarah Terez Malka, USA

Nicky Mansfield, USA ${ }^{\dagger}$

Carine Marks, South Africa

Brett Allen Matzek, USA ${ }^{\dagger}$

Ioannis Mavridis, Greece

Todd M. McGrath, USA

Omer Mei-Dan, USA

Christine Mermier, USA

Brendan H.A. Milliner, USA ${ }^{\dagger}$

Ajay Mishra, India

Ganesh Mohan, India

Wuelton Monteiro, Brazil

Matthew L. Moorman, USA ${ }^{\dagger}$

Damian Morgan, Australia

Audry Morrison, UK

Roger B. Mortimer, USA

David Murdoch, New Zealand

Andrew T. Nathanson, USA

Cristián Patricio Navarrete-Dechent, Chile

Maria Nemethy, USA

Martin Georg Niedermeier, Austria

Matias Nochetto, USA

Sean Nordt, USA

Robert Lee Norris, USA

Andrew Nyberg, USA

Gavarry Olivier, FRANCE

Gerald O'Malley, USA

Kathy Orloski, USA

Edward Joseph Otten, USA*

Peter Paal, Austria

Prativa Pandey, Nepal

Avinash Patil, USA
Joseph Pavelites, USA

Andre Michael Pennardt, USA

Patrick Peters, Luxembourg

Dan D. Petersen, USA

Lara Phillips, USA

Robin Pope, USA

Matiram Pun, Nepal*

Robert H. Quinn, USA

Saumitra Rege, USA

Aaron Reilly, USA

Dabor Resiere, France

Mara Fernandes Ribeiro, Brazil

George W. Rodway, USA*

Terry Rolan, USA

Lauren Rosenblum, USA ${ }^{\dagger}$

Steven Roy, Canada

Brent Ruby, USA

Anne-Michelle Ruha, USA

David A. Rust, USA

Emily Sagalyn, USA

Roy Salgado, USA

Mona Sarfaty, USA

Kathleen D. Saxon, USA

Courtney L Scaife, USA

Volker Schöffl, Germany*

Steven Gremel Schauer, USA ${ }^{\dagger}$

Sam Schimelpfenig, USA

Tod Schimelpfenig, USA

Andrew Schmidt, USA

Corinna Ariane Schoen, Switzerland

Robert Schoene, USA

Walter Schrading, USA

Andreas Schweizer, Switzerland

Thomas Seibert, USA ${ }^{\dagger}$

Alison Sheets, $\mathrm{USA}^{\dagger}$

David R. Shlim, USA

William R. Smith, USA

Simon Smith, Australia
Benji Smith, USA ${ }^{\dagger}$

Susanne J. Spano, USA

Rom Stevens, USA

Brian Mitchell Strickland, USA

Gage Alexander Stuntz, USA ${ }^{\dagger}$

Guenther Sumann, Switzerland

Douglas Sward, USA

Choo Hock Tan, Malaysia

Christopher Tedeschi, USA*

Saravanan Thangamani, USA

Martha Caperton Tissot van Patot, USA

Morgan Torris, $\mathrm{USA}^{\dagger}$

Angelika Underwood, USA ${ }^{\dagger}$

Jared Vagy, USA

Jiri Valenta, Czech Republic

Karen Van Hoesen, USA

Abraham Vivek, USA ${ }^{\dagger}$

Scott Votey, USA

Rob Walker, New Zealand

Jessica Walrath, USA ${ }^{\dagger}$

Matthew Ward, USA

Christine Warner, USA

Ian Wedmore, USA

Lori Weichenthal, USA

Eric Weiss, USA

Nicole Williams, USA ${ }^{\dagger}$

Christopher Williams, $\mathrm{USA}^{\dagger}$

F. Michael Williams-Bell, Canada

Bryan Wilson, USA

Matthew Wilson, USA

Jeremy S. Windsor, UK

Michael Yaron, USA

Cedric Yoshimoto, USA

David Young, USA

Ivan Yue, USA

Ken Zafren, USA* 